\title{
Nouvel éclairage sur les Acadiens à Belle-île-en- Mer à la fin du XVIIIe siècle
}

New Light on Acadians in Belle-Île-en-Mer at the End of the Eighteenth Century.

\section{(2) OpenEdition}

\section{Journals}

\section{Édition électronique}

URL : http://journals.openedition.org/abpo/2782

DOI : $10.4000 /$ abpo.2782

ISBN : 978-2-7535-3513-8

ISSN : 2108-6443

Éditeur

Presses universitaires de Rennes

Édition imprimée

Date de publication : 30 juin 2014

Pagination : 107-121

ISBN : 978-2-7535-3511-4

ISSN : 0399-0826

\section{Référence électronique}

Jean-Paul Moreau, « Nouvel éclairage sur les Acadiens à Belle-Île-en-Mer à la fin du XVIIle siècle »,

Annales de Bretagne et des Pays de l'Ouest [En ligne], 121-2 | 2014, mis en ligne le 30 juin 2016, consulté le 30 avril 2019. URL : http://journals.openedition.org/abpo/2782 ; DOI : 10.4000/abpo.2782 


\title{
Nouvel éclairage sur les Acadiens à Belle-Île-en-Mer à la fin du XVIII ${ }^{\mathrm{e}}$ siècle
}

\author{
Jean-Paul MOREAU \\ Président de la Société polymathique du Morbihan, Docteur en médecine, \\ titulaire d'un master d'histoire ${ }^{1}$
}

L'implantation de Français en Acadie fut contestée dès 1613 par les Anglais établis en Virginie. Une nouvelle "guerre de Cent Ans " américaine commença et se solda par 27 années d'occupation anglaise avant la cession définitive de l'Acadie péninsulaire à la Grande-Bretagne en 1713. Lors de la guerre de Succession d'Autriche, les Acadiens, considérés jusqu'alors comme des Français neutres, devinrent des rebelles en puissance. En 1755, leur refus de prêter serment d'allégeance sans réserve décida de leur déportation massive dans les colonies anglaises d'Amérique du Nord. La Virginie refoula environ 1200 de ces déportés dont 1048 survivants furent internés en Angleterre. Quatre ans plus tard, en pleine guerre de Sept-Ans, 3500 Acadiens furent envoyés en France dans le cadre d'un échange de prisonniers. En 1763, 778 Acadiens en provenance d'Angleterre débarquèrent ainsi à Morlaix et à Saint-Malo. Le gouvernement français était indécis quant au sort à réserver à ces réfugiés. Quelques dizaines de familles acceptèrent un transfert dans des colonies d'Outre-mer. Pour la plus grande majorité, il fallut envisager une implantation en terre de France. Choiseul s'enquit des disponibilités territoriales pour un accueil éventuel. Divers projets ne connurent pas de suite. Belle-Île-en-Mer, occupée entre 1761 et 1763 par les Anglais, fut restituée à la France en $1763^{2}$. Les États de Bretagne venaient de proposer l'attribution de terres en friche aux paysans bellilois selon la procédure de l'afféagement. Ils offrirent d'accueillir, dans ce cadre, des familles de réfugiés acadiens. En 1765, 57 familles acadiennes de Morlaix et 21 de Saint-Malo acceptèrent de se lancer dans cette entreprise. En vue de favoriser leur intégration, ces familles furent disséminées dans 40 villages des paroisses de lîle ${ }^{3}$.

1. MoREAU, Jean-Paul, Démographie et intégration des Acadiens installés à Belle-Île-en-Mer en 1765, Université de Bretagne-sud, mémoire de master, 2012.

2. CÉRINo, Christophe, «Enjeux stratégiques et opérations navales britanniques en Bretagne-Sud au XVII" siècle ", Annales de Bretagne et des Pays de l'Ouest, n 114-4, Rennes, PUR, 2007, p. 133-148.

3. Arch. dép. d'Ille-et-Vilaine, C 5156, 5157 et 5158. 
La question de leur devenir est controversée. Dominique Guillemet ${ }^{4}$ rappelle que « dès 1780 , les premières synthèses historiques sur Belle-Île conclurent à l'échec de l'installation. [...] Le bilan démographique est resté incertain [...] jusqu'aux années 1960 ». Les conclusions erronées d'Ernest Martin ${ }^{5}$ (1936) furent reprises dans les publications ultérieures. Il fallut attendre les travaux de Dominique Guillemet (1994) et de Christophe Cérino ${ }^{6}$ (2003) pour démontrer la relative réussite de l'intégration d'une fraction de la population acadienne belliloise. Dans sa thèse (2005), Jean-François-Mouhot ${ }^{7}$ conclut : "Au-delà des querelles de chiffres, il semble que le greffon acadien n'ait que partiellement pris ". L'analyse de l'historiographie révèle en effet d'importantes divergences des données statistiques. René Daligaut ${ }^{8}$, se référant à un document daté de 1791, dénombre la présence de 50 familles acadiennes sur les terres afféagées entre 1766 et 1768 . Le bulletin $n^{\circ} 40$ de l'Association pour l'histoire de Belle-Ille-en-Mer estime qu'entre douze et seize familles acadiennes sont restées à Belle-Île-en-Mer. Dominique Guillemet ${ }^{9}$ rappelle les chiffres avancés par Jean Ogée (20), A. Detaille (32), F.-M. Cayot-Delandre (12) et Ernest Martin (16). Lui-même opte pour les deux tiers soit 48.

Or, ces évaluations ne sont pas étayées par des données numériques dont la fiabilité a été validée. Ceci explique la très grande différence entre les valeurs extrêmes qui vont du simple (12) au quadruple (50). Dans le cadre de notre master de recherche ${ }^{10}$, nous avons eu pour objectif d'apporter une réponse susceptible de clarifier, par la méthode des statistiques, les discussions concernant ces querelles de chiffres en nous basant uniquement sur des données factuelles et numériques fiables et aussi exhaustives que possible. Nous avons eu pour sources les archives départementales d'Ille-et-Vilaine $^{11}$, du Morbihan ${ }^{12}$, de Loire-Atlantique ${ }^{13}$ et de la Vienne ${ }^{14}$.

4. GulLLEMET, Dominique, "Les Acadiens de Belle-Île-en-Mer : légende noire et histoire en (re)-construction ", Acadiens, mythes et réalités, Études acadiennes, université de Moncton, vol. 37, déc. 1994, p. 127-144.

5. MARTIN, Ernest, Les exilés acadiens au XVIII siècle et leur établissement en Poitou, Paris, Hachette, 1936.

6. CÉRINO, Christophe, «Les Acadiens à Belle-Île-en-Mer : une expérience originale d'intégration en milieu insulaire à la fin du XVIII ${ }^{\mathrm{e}}$ siècle ", Annales de Bretagne et des Pays de l'Ouest, n' 110-1, 2003, p. 115-124.

7. Mоuнот, Jean-François, Les réfugiés acadiens en France (1758-1785) : l'impossible réintégration?, Thèse de doctorat d'Histoire et Civilisation, Institut universitaire de Florence, octobre 2005. Québec, Éditions du Septentrion, 2009.

8. Daligaut, René, "Les Acadiens en Bretagne : ceux de Belle-Île-en-Mer ", Cahiers de l'Iroise, n $^{\circ}$ 3, juil.-août 1954, p. 24-29.

9. Guillemet, Dominique, op. cit.

10. MoreaU, Jean-Paul, op. cit.

11. Arch. dép. d'Ille-et-Vilaine, série $C$ : Intendance et États de Bretagne, particulièrement C 5136, 5158, 6176.

12. Arch. dép. du Morbihan, série 4A : Domaine royal de Belle-Île-en-Mer 4A 49, série 4E: Registre paroissiaux de 1751 à 1792 (baptêmes, mariages, sépultures), série 6E (de 15475 à 15484) : archives notariales de Maître J.-B. Thébault de 1766 à 1785.

13. Arch. dép. de Loire-Atlantique, Fonds de l'Inscription maritime, C 1287 et 1288.

14. Arch. dép. de la Vienne, Chartrier de la Roche de Bran série 41J 124-3 et 2J dép. 22 art. 124-1. 
L'ampleur du travail sur les registres d'état civil nous a convaincu d'avoir recours aux données de l'état civil du très important corpus établi à l'aide du logiciel Heredis par l'association Racines et Rameaux des Français d'Acadie. Il était cependant indispensable de valider le degré de fiabilité des données ainsi recueillies. Il a donc été réalisé une vérification complète de toutes les données d'état civil en consultant les registres des quatre paroisses de l'île de 1765 à 1785. Les données du logiciel Heredis ont été confirmées dans $96,1 \%$ des cas, les défauts de concordance ne relevant pas d'erreurs mais d'imprécisions concernant le jour ou le mois d'un événement ${ }^{15}$. Ces résultats ont permis de conclure à la très bonne fiabilité des données recueillies par ce logiciel. Nous avons en outre exploité les dates et lieux de naissance des déclarations de généalogie de $1767^{16}$ ainsi que les archives notariales belliloises de 1766 à $1785^{17}$, données d'archives méconnues par les publications antérieures. L'ensemble des données recueillies permet d'apporter un nouvel éclairage sur l'intégration des Acadiens de Belle-Île-en-Mer en nous appuyant sur des données factuelles et numériques des plus fiables.

\section{Les marqueurs identitaires, obstacles surmontables de l'intégration}

L'installation en 1765 de 363 étrangers acadiens à Belle-Île-en-Mer était une opération délicate car il s'agissait de faire cohabiter deux populations réputées à fortes caractéristiques identitaires ${ }^{18}$. La population belliloise s'élevait alors à environ 5000 habitants répartis entre des ruraux occupant 127 villages et des citadins (environ 950) habitant Le Palais. Les ruraux agriculteurs avaient vécu dans l'univers du contrôle étroit du monde féodal et partageaient une culture et une langue bretonne. Repliés sur leurs terres agricoles, leur sentiment d'appartenance à un groupe se limitait à l'unité villageoise. Il n'est pas démontré qu'ils aient entretenu de forts sentiments d'appartenance à leur île. Les Palantins, par contre, étaient en contact depuis plus d'un siècle avec des militaires et des fonctionnaires royaux issus de différentes provinces françaises. Ils s'adonnaient à des activités commerciales et maritimes et utilisaient la langue française. Les Acadiens, sujets britanniques depuis 1713, étaient des ruraux adeptes de la libre entreprise qui avaient appris à composer avec leurs gouvernants anglais. Ils étaient fortement solidaires en raison de leurs liens familiaux. Le peuplement de l'Acadie résultait du taux élevé de fécondité d'un nombre

15. Nous tenons à remercier M. André Thomas, vice-président de l'association Racines et Rameaux des Français d'Acadie, qui nous a ouvert sa base de données d'état civil.

16. Arch. dép. du Morbihan, série 4 A 49.

17. Arch. dép. du Morbihan, série 6 E de 15475 à 15484.

18. Voir à ce sujet : CÉRINo, Christophe, "L'îlien, la guerre et l'étranger : quelques observations sur la perception de l'autre à Belle-Île-en-mer au XVIII siècle ", Mémoires de la Société d'archéologie et d'histoire de Bretagne, n ${ }^{\circ}$ 89, 2011, p. 291-300; CÉRINo, Christophe, "Les paysans de la mer et l'événement : destructions, misères et reconstruction des campagnes au lendemain de la guerre de Sept-Ans ", Enquêtes rurales, n 7, Caen, Presses universitaires de Caen, 2000. 
initial restreint de familles. Plus de la moitié des arrivants se répartissaient entre seulement cinq patronymes et 66 familles sur 78 (85\%) étaient originaires du Bassin des Mines de l'Acadie péninsulaire. Ces familles, déportées vers la Virginie puis refoulées vers l'Angleterre en 1755, avaient été débarquées en 1763 à Morlaix et Saint-Malo. Parmi ces déportés de 1755, quatorze étaient nés en Acadie avant 1713 et 237 entre 1713 et $1755 ; 46$ étaient nés en Angleterre entre 1755 et 1763 et 66 en France entre 1763 et 1765 . Les 297 sujets britanniques, qui s'étaient jusqu'alors considérés comme des "Français neutres ", devenaient des sujets du roi de France au statut de réfugiés.

Sur le plan démographique cette population acadienne se démarquait de la population française de l'époque. La distribution des classes d'âge ${ }^{19}$ révèle que les moins de 35 ans représentaient $83 \%$ contre $65 \%$ dans la population française ${ }^{20}$. La surnatalité, après l'arrivée en France, a compensé une surmortalité des enfants nés pendant les années de déportation. Il existe de même une surmortalité des classes d'âge supérieures à 35 ans. Ces anomalies démographiques s'expliquent par le fait que les âges extrêmes de la vie ont été les plus exposés aux facteurs de mortalité aggravés par les perturbations sociales. Il en résulte que la population acadienne belliloise est une population singulièrement jeune, les perturbations sociales ayant opéré une sélection des sujets les plus résistants.

Pour Dominique Guillemet ${ }^{21}$, il n'existait cependant pas "d'identités irréductibles " entre les deux populations. Dans les deux cas en effet il s'agissait de ruraux catholiques pratiquants et respectueux du système monarchique de droit divin. C'est le monde rural bellilois qui fut touché par l'implantation des réfugiés acadiens. Les îliens ruraux bénéficièrent, comme les Acadiens, de l'attribution d'afféagements. Il y eut une égalité approximative de répartition des terres tant entre les Acadiens qu'entre les Acadiens et les Bellilois ${ }^{22}$. La nécessité pour tous de s'atteler à des travaux de reconstruction favorisa le rapprochement. En effet pendant l'occupation anglaise de l'île (1761-63) plus de 60 \% des habitations rurales avaient été entièrement ou partiellement détruites par les occupants pour se procurer du bois de chauffage ou de construction ${ }^{23}$. Les deux populations rurales, de même statut social et confrontées aux mêmes travaux agricoles, devaient cependant franchir la barrière linguistique. En réalité une bonne partie des ruraux autochtones comprenait le français à défaut de le parler. À titre de confirmation soulignons que " le clergé rédige tous ses actes en français. Les gens de robe aussi. Toutes les bannies (publications) [...] précisent que la lecture [a été] faite à la sortie de la messe [...] en langue française ${ }^{24}$ ".

19. MoreAU, Jean-Paul, op. cit., p. 39.

20. D'après la pyramide des âges en France en 1750, Population et sociétés (bulletin de l'INED), n 346 , mai 1999.

21. GuILLEMET, Dominique, op. cit.

22. Ibidem.

23. Arch. dép. Ille-et-Vilaine, C 5126.

24. Bulletin de l'association pour l'histoire de Belle-Île-en-Mer, $\mathrm{n}^{\circ}$ 5, janvier-mars 1965, p. 9. 
Selon le spécialiste de la démographie historique Jacques Dupâquier ${ }^{25}$ le mariage est le principal moteur de l'intégration. Pour les Acadiens bellilois, ce sont les mariages mixtes qui témoignent que les marqueurs identitaires divergents des deux communautés ont été rapidement et massivement surmontés : sur 42 mariages célébrés entre 1765 et 1785 , on compte déjà 36 mariages mixtes (85,7 \%). Parmi les Acadiens arrivés à Belle-lle-en-Mer en 1765 ou nés à Belle-Île-en-Mer entre 1765 et 1785 , nous avons dénombré ${ }^{26}$, entre 1766 et 1815, un total de 113 mariages (106 célébrés à Belle-Île-enMer, cinq à Auray et deux à Lorient). Seuls quinze de ces mariages sont des mariages entre Acadiens. A contrario, 98 soit 86,7 \% sont des mariages mixtes.

Tableau 1 - Distribution des mariages interacadiens et mixtes

\begin{tabular}{|l|l|l|l|}
\hline \multicolumn{2}{|l|}{} & Nombre & Pourcentages \\
\hline Mariages interacadiens & Belle-Île-en-Mer & 15 & $13,3 \%$ \\
\hline \multirow{4}{*}{$\begin{array}{l}\text { Mariages mixtes : } \\
\text { Origine des conjoints }\end{array}$} & Ports et autres du Morbihan & 51 & $45,1 \%$ \\
\cline { 2 - 4 } & France : en poste à Belle-Île & $16^{*}$ & $14,2 \%$ \\
\cline { 2 - 4 } & France : autres & 11 & $17,7 \%$ \\
\hline TOTAUX & 113 & $9,7 \%$ \\
\hline
\end{tabular}

* Auray : 5, Lorient : 2, Carnac : 2, Quiberon : 1, Crac'h : 1, Autres : 5

** Militaires : 14 - Employés civils : 4 - Douaniers : 2

Il ne s'agit pas d'un choix par défaut. En effet, au sein des 98 couples mixtes, on dénombre 42 garçons acadiens et 56 filles acadiennes qui auraient très bien pu convoler entre eux. Il n'en fut rien. Deux facteurs majeurs ont contribué au succès des mariages mixtes. La dispersion des familles acadiennes dans 40 villages des quatre paroisses favorisa un contact étroit avec les résidants bellilois par le biais de travaux ruraux, de participations aux activités paroissiales et de démarches diverses dans les bourgs.

Cependant, cette dispersion ne peut à elle seule expliquer les 98 mariages avec des conjoints originaires de Belle-Île-en-Mer ou y résidant. Il fallait en effet franchir des barrières culturelles dont la langue et les éventuels hiatus de statut social. D'où le rôle du second facteur en faveur des mariages mixtes à savoir la volonté d'intégration des parents acadiens de Belle-Île-enMer. À cette époque, en effet, le choix du conjoint relevait des accommodements et des décisions des parents des deux familles et non d'une cooptation des intéressés. Ainsi la volonté d'intégration des parents acadiens

25. DUPÂQUIER, Jacques, La population française aux XVII et XVII ${ }^{e}$ siècles, Paris, PUF, coll. "Que sais-je? », 1979.

26. MOREAU, Jean-Paul, op. cit., p. 42. 
est manifeste. Nous avons vu que pour Dominique Guillemet ${ }^{27}$ il n'existait pas d'identités irréductibles entre Bellilois et Acadiens. Toutefois, pour des motifs divers, il existe au sein d'une diaspora un très large gradient en ce qui concerne la volonté de s'intégrer. Il n'est donc pas surprenant que certains couples ou isolés acadiens aient éprouvé des difficultés à envisager avec optimisme leur avenir à Belle-Ille-en-Mer. La grande majorité des départs se situa entre 1766 et 1785 . Il faut toutefois étudier séparément les départs de la première décennie et ceux de la seconde. En effet, en application de leurs contrats d'afféagement, les contractants ne furent autorisés à vendre, aliéner ou hypothéquer leurs concessions qu'à partir du premier janvier 1776 .

\section{Départs de la première décennie (1766-1775) : vers la France continentale}

Les deux premiers départs intervinrent dès 1767. Le 9 novembre, l'afféagiste $n^{\circ} 20$ " fait exponse " devant notaire en se déclarant " dans l'impossibilité [...] de pouvoir exploiter dans l'état où elle est (sa) portion de terre ". Le 3 décembre 1967, le procès-verbal d'un commissaire de Bretagne ${ }^{28}$ rapporte que l'afféagiste $n^{\circ} 17$ est parti pour Saint-Malo en vue de " rejoindre son frère et sa sœur ". Après ces deux premiers départs, les premières récoltes intervinrent au cours de l'année suivante, 1768. Les indemnités journalières furent versées jusqu'en décembre 1769 et, à partir de 1770, chaque propriétaire fut soumis au versement d'une rente foncière et d'une dîme. C'est dans ce contexte que les correspondances de 1769 du receveur de Kermarquer $^{29}$, nous informent de cinq départs ${ }^{30}$. Les actes notariés ${ }^{31}$ font état de cinq autres départs avant $1772^{32}$.

Au cours de l'année 1772, un recensement des Acadiens, signé par le commissaire général de la Marine Lemoyne, fut réalisé en vue de la création d'un établissement en Poitou ${ }^{33}$. En ce qui concerne les Acadiens de Belle-Île-en-Mer, le récapitulatif en décompte 103. Ce rôle des familles des Acadiens dits de Belle-Île inclut 22 Acadiens non bellilois. Ce recensement ne concerne donc que 81 Acadiens vraiment bellilois dont 19 seulement résident encore à Belle-Île-en-Mer. Les 62 autres appartiennent à 14 familles qui ont quitté l'île et dont le lieu de résidence est précisé. Au total, au cours de la première décennie, les départs définitifs dûment documentés concernent seize familles comptant 86 individus auxquels il faut ajouter huit personnes isolées soit un total de 94 individus $^{34}$.

27. GUILLEMET, Dominique, op. cit.

28. Arch. dép. Ille-et-Vilaine, série C 5149.

29. Ibidem.

30. Afféagistes $\mathrm{n}^{\circ} 28$ (perte de ses bestiaux), $\mathrm{n}^{\circ} 55$ et 61 (affermage), $\mathrm{n}^{\circ} 62$ (exponse),

$\mathrm{n}^{\circ} 77$ (départ pour Nantes).

31. Arch. dép. du Morbihan, série 6 E 15475 à 15484.

32. Afféagistes $n^{\circ} 10$ (incendie), $n^{\circ} 51$ et 53 (exponses), n ${ }^{\circ} 22$ et 68 (affermages).

33. Arch. dép. de la Vienne, Chartrier de la Roche-de-Bran, série 41 J 124-3.

34. MorEAU, Jean-Paul, op. cit., p. 56-57. 
Quelles furent les motivations de départ de ces seize familles acadiennes avant l'échéance de dix ans prévue au contrat? S'agit-il uniquement ou majoritairement de motifs économiques comme le rapporte une large majorité des publications antérieures? Les deux départs de l'année 1767 ne sont manifestement pas économiques. L'un est un regroupement familial. L'autre, sans explication objective, relèverait d'une impossibilité d'exploiter les terres attribuées et ceci avant les premières semailles. Pour les départs situés entre 1768 et 1772, la quasi-totalité des auteurs se réfère aux explications du gouverneur de Warren. Il écrit le 31 octobre 1772 à l'abbé Joseph Marie Terray ${ }^{35}$, contrôleur général des finances du roi. "Les mauvaises récoltes que les colons ont essuyées depuis trois ans et le prix excessif des grains [...] ont épuisé le peu de ressources qu'ils avaient [...]. Une bonne partie des Acadiens sont forcés et se disposent à abandonner lîle. "Une correspondance du commissaire général Lemoyne ${ }^{36}$ reprend, sans enquête particulière, les thèses du gouverneur de Warren. Cependant l'intégration matérielle et économique semble déjà bien avancée : l'inventaire des biens fait lors du décès en 1772 de l'afféagiste $\mathrm{n}^{\circ} 14$ se montait à 650 livres $^{37}$. En réalité les explications du gouverneur de Warren ne concordent pas avec les faits. D'évidence les deux premiers départs de 1767 n'y répondent pas. Les sept départs suivants sont survenus en 1768 et $1769^{38}$. Deux de ces départs s'expliquent l'un par la perte du bétail, l'autre par un incendie. Les autres afféagistes ont fait exponse ou ont affermé leurs terres sans fournir d'explication. À partir de 1770 les afféagistes cessent de toucher les indemnités journalières et sont tenus de verser une redevance annuelle. Il est évident qu'ils ont exprimé leur contrariété aux autorités locales, d'où les correspondances du gouverneur (mais seulement trois ans plus tard) et ses prédictions alarmistes sur des départs imminents. En réalité, au cours des trois ans 1770-1772, il n'y a eu que trois départs ${ }^{39}$ et au cours des trois années suivantes, il n'y en eut que deux ${ }^{40}$.

Au total, sur les seize départs de la première décennie, deux ne sont manifestement pas économiques et deux le sont manifestement. Les autres départs exigent que soient recherchées des données factuelles au lieu de se baser uniquement sur les seules opinions exprimées par le gouverneur de Warren qui doivent être atténuées. Primo : Ces opinions sont exprimées trois années après la convergence de la nécessité de payer une redevance et de ne plus percevoir des indemnités journalières. Il est évident qu'elles sont le reflet des récriminations (compréhensibles) des afféagistes acadiens. Secundo : Le baron de Warren, en 1772, est âgé de 67 ans. C'est un vieil homme usé et un peu découragé (il décédera trois ans plus tard). La disparition en 1772 de son appui indéfectible, l'abbé Le Loutre, l'a contraint

35. Arch. dép. du Morbihan, série E art. 1464-2.

36. Bibliothèque municipale de Bordeaux, Ms 1480, $\mathrm{f}^{\circ}$ 469-471.

37. CÉRINO, Christophe, op. cit.

38. Familles $\mathrm{n}^{\circ} 28,55,61,62,77$ (de Kermarquer) et $\mathrm{n}^{\circ} 10$ et 51 (Maître Thébault).

39. $\mathrm{N}^{\circ} 53$ en 1770,68 en 1771 et 22 en 1772.

40. № 16 en 1773 et 30 en 1774. 
à en appeler, en vain, au contrôleur général des finances. On ne saurait lui reprocher un certain pessimisme. Cependant, ni la météorologie, ni la géologie, ni la pédologie ne peuvent rendre compte de la répartition aléatoire des villages affectés par des départs. On peut par contre rapprocher ces départs des données suivantes : huit familles venues de Morlaix et deux de Saint-Malo ${ }^{41}$, soit dix sur seize (62,5\%) sont retournées dans ces ports où elles avaient de nombreux parents ${ }^{42}$. Il est donc tout à fait crédible de penser que dans les motivations de départ s'est immiscée une importante composante impliquant des phénomènes d'interdépendance et/ou d'interaction relevant de ces liens familiaux et donc de souhaits spontanés ou suggérés de regroupement familiaux.

Ainsi, on peut avancer, sans trop de risque d'erreurs, que, au cours de la première décennie, le nombre de départs réellement ou vraisemblablement liés à des motifs économiques ne dépasse pas sept ${ }^{43}$. Quant aux neuf autres départs, nous ne pouvons nous prononcer. Ce qui est certain, c'est que ces familles ont quitté Belle-Île-en-Mer parce qu'elles avaient le sentiment qu'elles pouvaient trouver mieux ailleurs en particulier dans les ports de Morlaix et de Saint-Malo où résidaient des familles acadiennes qui leur étaient apparentées et dont elles partageaient l'esprit de solidarité et d'entreprise.

\section{Départs de la seconde décennie (1776-1785) : vers la Louisiane et la France continentale}

Deux dates de l'année 1776 jouèrent un rôle majeur dans la destinée des Acadiens bellilois : d'une part le premier janvier, date à laquelle les contractants pouvaient vendre, aliéner ou hypothéquer leurs concessions, d'autre part le 4 juillet, jour de l'adoption par le Congrès des représentants des treize colonies anglaises d'Amérique du Nord de la " Déclaration d'Indépendance " qui déclencha la guerre d'Indépendance (1776-1783). Depuis 1763, de nombreux Acadiens, déportés et mal accueillis dans les colonies anglaises, tentaient avec un certain succès de rejoindre des territoires francophones d'Amérique du Nord comme le Canada ou l'Acadie de terre ferme en dépit de leur occupation par des troupes britanniques. D'autres Acadiens déportés optèrent pour la Louisiane francophone à l'ouest du Mississipi (cédée à l'Espagne par le traité de 1763). Les Acadiens de France n'étaient pas démunis d'informations concernant une telle réalité. Lors des déclarations de généalogie à Belle-Île-en-Mer en $1767^{44}, 25$ chefs de famille furent en mesure de donner des informations sur des familles entières ou des membres isolés de leur parenté dispersés outre-Atlantique. En dépit de

41. Morlaix : $\mathrm{n}^{\circ} 20,22,47,55,61,62,77$; Saint-Malo : $\mathrm{n}^{\circ} 17,68$.

42. MoreaU, Jean-Paul, op. cit., p. 56.

43. $\mathrm{N}^{\circ} 10$ (incendie), $\mathrm{n}^{\circ} 28$ (perte du bétail), $\mathrm{n}^{\text {os }} 22,53$ et 68 (départs entre 1770 et 1772 ), $\mathrm{n}^{\text {os }} 16$ et 30 (départs en 1773 et en 1774).

44. Arch. dép. du Morbihan, série 4 A 49. 
leurs tribulations pendant une décennie, les Acadiens bellilois détenaient des informations dont le degré de précision laisse à penser que des messages transatlantiques émanant de la diaspora acadienne nord-américaine parvenaient aux Acadiens réfugiés en France. Certains de ces messages confirmaient des réussites de tentatives de gagner des territoires nordaméricains francophones, Canada et Louisiane. Jean-François Mouhot ${ }^{45}$ rapporte que les réfugiés acadiens " échangeaient une intense correspondance [...] à travers la Manche et l'Atlantique ». L'expiration du délai de dix ans des contrats d'afféagement et l'information du déclenchement de la guerre d'Indépendance expliquent la seconde vague de départs d'Acadiens de Belle-Île-en-Mer. Les archives notariales sont à ce sujet des plus éloquentes. En majorité les afféagistes candidats au départ signèrent des contrats de ventes définitives de leurs concessions "pour toujours et à jamais. " Un petit nombre toutefois se contenta plus prudemment d'affermer leurs terres pour une durée déterminée.

Les départs au-delà de 1775 sont survenus dans un contexte socioéconomique très différent de celui de la première décennie. Les candidats au départ avaient mis leurs terres en valeur pendant dix années ou plus. Ces exploitations agricoles avaient atteint ou dépassé le seuil de rentabilité. Ainsi valorisées, elles purent être vendues dans de bonnes conditions comme nous le confirme les contrats de vente des archives notariales ${ }^{46}$. Les concessions ont trouvé des repreneurs dans un bref espace de temps. Vingt contrats de vente ${ }^{47}$ et sept contrats d'affermage ${ }^{48}$ firent l'objet d'actes notariés ${ }^{49}$ entre 1776 et 1785 . L'intégration économique pour certains Acadiens bellilois était bien réelle. Précisons que cinq des concessions acadiennes mises en vente à partir de 1776, ont été achetées par des Acadiens dont les familles sont par la suite restées définitivement à Belle-Ile-en-Mer ${ }^{50}$. La succession de l'afféagiste ${ }^{\circ} 2$ s'élevait en 1785 à 1288 livres $^{51}$. Ce ne sont donc pas des difficultés économiques ni de simples souhaits de regroupement familiaux qui ont motivé les départs de la seconde décennie. Il faut distinguer ceux qui partirent en Louisiane et ceux qui, ayant quitté BelleÎle-en-Mer, restèrent en France continentale.

Les destinations très majoritaires des départs de la seconde décennie furent Nantes, Chantenay et Paimbœuf. Quelques partants sans doute y ont retrouvé certains parents mais pour la plupart, après la vente ou l'affermage de leurs concessions, il s'agissait de pouvoir partager avec les 1400 res-

45. Mouнoт, Jean-François, op. cit., p. 320.

46. Arch. dép. du Morbihan, série 6 E 15478 à 15484 (1775 à 1785).

47. Trois en 1776 (familles $\left.n^{\circ} 36-37-65\right)$, dix en 1777 ( $n^{\circ}$ 5-18-23-38-45-49-57-67-73), un en $1778\left(n^{\circ} 41\right)$, un en $1779\left(n^{\circ} 7\right)$, deux en $1780\left(n^{\circ} 20-29\right)$, un en $1783\left(n^{\circ} 76\right)$, un en 1784 $\left(n^{\circ} 15\right)$ et un en $1785\left(n^{\circ} 40\right)$.

48. Familles n ${ }^{\circ}$ 26-27-31-59-63-71-74.

49. MoreAU, Jean-Paul, op. cit., p. 59-61.

50 . Familles $n^{\circ} 6,14,56$ et 70 .

51. Famille ${ }^{\circ} 2$, CÉRINO, Christophe, op. cit. 
capés de l'échec du Poitou ${ }^{52}$, repliés sur Nantes, l'espoir d'un retour en Amérique. En effet, ces trois ports connaissaient un trafic transatlantique permanent, en particulier pour approvisionner en armes et en matériel les insurgés nord-américains. En 1772, des Acadiens avaient envoyés au ministre une pétition pour passer en Louisiane ${ }^{53}$. Une correspondance du duc de Pérusse ${ }^{54}$ mentionne que " les Acadiens étant parvenus à se réunir en corps de nation, il leur sera plus facile d'obtenir [...] la permission de passer en Louisiane ". À Nantes, le subdélégué de l'intendant de Bretagne ${ }^{55}$ rend compte " qu'un perturbateur réclame d'aller en Louisiane où sont ses parents ". Une vingtaine de familles se joignirent à lui pour appuyer sa demande.

La signature du traité de paix de Paris en 1783 va permettre la concrétisation de ces espoirs. Au sein des diverses communautés acadiennes circula une pétition demandant au roi d'Espagne de leur accorder la permission de s'y rendre. Charles III d'Espagne accéda à cette requête et Vergennes, ministre des Affaires étrangères, réussit à convaincre Louis XVI. En 1785, sept navires prirent à leur bord, selon les listes d'embarquements, 1594 Acadiens de France pour les transporter en Louisiane ${ }^{56}$ et 57 . Parmi ceux-ci, un décompte méticuleux, précisant pour chaque famille et pour chaque isolé les numéros exacts sous lesquels ils ont été inscrits sur les listes d'embarquements ${ }^{58}$, nous a permis de dénombrer 21 familles et treize isolés totalisant 132 émigrants acadiens bellilois. Au total, les émigrants bellilois officiellement embarqués en 1785 se répartissent ainsi : trois familles et trois isolés totalisant seize émigrants qui avaient quitté Belle-Ile-en-Mer avant 1776 et que nous avons comptabilisés dans les départs de la première décennie et dix-huit familles et dix isolés totalisant 116 émigrants qui ont quitté Belle-Île-en-Mer après 1776 et que nous comptabilisons dans les départs de la seconde décennie. D'autres Acadiens bellilois, au nombre de quinze, sont partis en Louisiane à titre personnel. Leurs dates d'embarquements se situent vers 1785. Les intéressés devaient séjourner un temps certain dans les ports d'embarquement avant de trouver une opportunité. Ils ont donc quitté Belle-l̂le-en-Mer avant 1785. Ceci est évident pour sept d'entre eux dont la vente ou l'affermage de la tenue familiale se situe entre 1772 et 1784 . Nous désignons ces émigrants, qui ont trouvé à s'embarquer par leurs propres moyens, sous le terme d'auto-embarquement $c a$ 1785. Nos recherches sur l'état civil des Acadiens bellilois nous ont permis de retrouver pour treize d'entre eux la date et le lieu précis de leur décès en

52. Braud, Gérard-Marc, De Nantes à la Louisiane, l'histoire de l'Acadie, l'odyssée d'un peuple exilé, Cholet, Éditions de l'Ouest, 1994.

53. Bibliothèque municipale de Bordeaux, Ms 1480, f $89-90$.

54. Arch. dép. de la Vienne 2 J dép. 22 art. 124-1.

55. Arch. dép. d'Ille-et-Vilaine, série C 6176.

56. Arch. dép. de Loire-Atlantique, Fonds de l'Inscription maritime, C 1287-1288.

57. HÉBert, Donald J., Acadian Families in Exile, 1785, Raynes Louisiana, Hébert Publications, 1995.

58. MorEAU, Jean-Paul, op. cit., annexe IV, p. 119-121. 
Louisiane. Quant aux deux autres nous savons seulement qu'ils sont décédés en Louisiane ${ }^{59}$. Les départs de la seconde décennie pour la Louisiane s'élèvent donc à 116 embarquements officiellement enregistrés en 1785 plus quinze auto-embarquements ca 1785 soit un total de 131. La somme des départs en Louisiane, en additionnant les départs de Belle-Île-en-Mer de la première et de la seconde décennie, est de $16+131=147$. La famille $\mathrm{n}^{\circ} 23$ s'étant réunie en Louisiane à la faveur d'un auto-embarquement $c a$ 1785 , le total des familles parties s'installer en Louisiane s'élève au nombre de 22. Ces 147 émigrants témoignent qu'au tréfonds de la mentalité de ces Acadiens, qui abandonnaient un certain niveau de confort bellilois pour une aventure non dénuée d'incertitudes, perdurait " un désir d'ailleurs " espéré meilleur ainsi qu'une volonté d'entreprendre. Ce désir d'ailleurs s'explique d'une part par la persistance des correspondances acadiennes transatlantiques et d'autre part par le mythe transculturel du « bon vieux temps ", avatar du mythe de l'âge d'or. Les réfugiés acadiens de France, incluant ceux de Belle-Île-en-Mer, étaient informés des péripéties de la guerre d'Indépendance nord-américaine.

Le mythe transculturel du " bon vieux temps ", surtout quand il concerne un ailleurs exotique, a toujours des sujets réceptifs au sein d'un auditoire. D. Guillemet ${ }^{60}$ pense que « chez les réfugiés acadiens de Belle-l̂le [...] s'il y a identité collective, elle est celle d'une diaspora pouvant générer à la fois chez ses membres désir éperdu de fixation ou au contraire un appel plus ou moins permanent au voyage ». C'est exactement ce que nous observons dans notre population acadienne belliloise. Sur les 363 Acadiens arrivés à Belle-Île-en-Mer en 1765, 251 étaient nés en Acadie (69 \%) dont 162 (46\%) avaient plus de neuf ans lors de la déportation de 1755, donc plus de dix-neuf ans en arrivant à Belle-Île-en-Mer. Auprès d'un tel auditoire, les récits familiaux et interfamiliaux ont certainement entretenu la mémoire de la terre natale, le plus souvent en l'embellissant. Selon la réceptivité de chacun, en particulier du chef de famille, le camaïeu des sentiments s'est déployé entre le désir de fixation et la nostalgie des grands espaces fertiles nord-américains. Tous les membres des familles passées en Louisiane ne participèrent pas à l'émigration outre-Atlantique. Certains étaient décédés avant les embarquements entre 1777 et 1785 : douze à Paimbœuf, sept à Nantes et sept à Chantenay soit un total de 26 individus ${ }^{61}$. D'autres membres de ces familles, au nombre de 18, ne suivirent pas leur parentèle émigrante ${ }^{62}$. En outre, tous les départs de Belle-Île-en-Mer au cours de la seconde décennie, ne furent pas liés à l'émigration vers la Louisiane. Les départs de 31 individus vers divers ports bretons sont authentifiés par des actes notariés ${ }^{63}$.

59. MoreaU, Jean-Paul, op. cit., p. 65 et annexe V p. 122 à 172.

60. Guillemet, Dominique, op. cit.

61. MoREAU, Jean-Paul, op. cit, p. 66.

62. Ibidem, p. 67.

63. Ibid. 


\section{Bilan des familles acadiennes demeurées à Belle-Île-en-Mer et en France continentale après 1785}

Aux départs, précisément documentés, des deux premières décennies, il faut ajouter treize familles (56 individus) qui ont quitté Belle-Îleen-Mer à des dates imprécises ou non connues entre 1766 et 1785 pour diverses destinations ${ }^{64}$. Sur la base des états de 1765 et 1766 des familles acadiennes débarquées puis installées dans des villages bellilois, états complétés par les déclarations de généalogie de 1767, nous avons établi une liste de 398 noms d'Acadiens, à savoir les 363 débarqués plus 35 nouveaunés ${ }^{65}$. Les données démographiques du logiciel Heredis complétées par nos propres recherches de l'état civil, sont venues enrichir cette liste par l'apport de 291 naissances ce qui nous donne un total de 689 noms $^{66}$. Cette liste recense les noms et les données de l'état civil des Acadiens débarqués en 1765 ainsi que ceux de leurs enfants et petits-enfants nés à Belle-Ille-enMer avant le 31.12.1785. L'étude de cette liste nous autorise à présenter le bilan complet des départs de l'île entre 1766 et 1785 .

\section{Tableau 2 - Bilan complet des départs de Belle-Île-en-Mer entre 1766 et 1785}

\begin{tabular}{|l|l|l|}
\hline \multirow{3}{*}{1766 à 1775} & Demeurés en France continentale & 78 \\
\cline { 2 - 3 } & Partis ensuite en Louisiane en 1785 & 16 \\
\hline \multirow{4}{*}{$\begin{array}{l}1775 \\
\text { à } \\
1785\end{array}$} & Embarqués pour la Louisiane en 1785 & 116 \\
\cline { 2 - 3 } & Auto-embarqués pour la Louisiane circa 1785 & 15 \\
\cline { 2 - 3 } & Membres des familles embarquées : décédés avant 1785 & 26 \\
\cline { 2 - 3 } & Membres des familles embarquées : restés en France & 18 \\
\cline { 2 - 3 } & Membres de familles demeurées en France (actes notariés) & 31 \\
\hline \multirow{2}{*}{ Total des départs 1785} & Partis pour le continent à des dates non précisées & 56 \\
\hline
\end{tabular}

Le décompte des Acadiens ayant quitté Belle-Île-en-Mer avant 1786 s'élève ainsi à 356 individus. Si l'on se rappelle le nombre de 363 arrivants en 1765 , un tel constat conduit à se poser la question de savoir combien d'Acadiens demeuraient dans l'île après ces départs. Ce décompte est en effet fondamental pour valider toute étude prosopographique du groupe social que représentent les Acadiens de Belle-Île-en-Mer en vue d'évaluer leur intégration au sein de la société belliloise. Notre liste des données de l'état civil des Acadiens installés ou nés à Belle-Île-en-Mer entre 1765 et 1785 comporte 689 noms ${ }^{67}$. Pour calculer le nombre d'Acadiens présents à Belle-

64. Ibid., p. 68-70.

65. MoReAU, Jean-Paul, op. cit, annexe II, p. 99-115.

66. Ibidem, annexe v, p. 122-172.

67. MoreAU, Jean-Paul, op. cit., annexe v, p. 122-172. 
Île-en-Mer au 31 décembre 1785, il nous faut soustraire de ce total général non seulement les 356 départs de l'île mais aussi les 109 décès qui y sont intervenus entre ces deux dates, ce qui donne : $689-(356+109)=224$.

En nous basant sur les lieux et dates des données de l'état civil, cette population de 224 individus, présents dans l'île à la fin de l'année 1785, se divise en deux groupes. Le premier groupe est celui des Acadiens installés en 1765 ou nés avant 1786 dont les dates et les lieux de décès à Belle-Îleen-Mer après 1785 sont précisément établis. Ce sont manifestement des Acadiens qui sont demeurés définitivement à Belle-Île-en-Mer. Leur nombre s'élève à 126 individus ${ }^{68}$. Le second groupe est celui des Acadiens installés en 1765 ou nés avant 1786 qui sont décédés à des dates et en des lieux connus hors de Belle-Île-en-Mer après 1785. Il s'y ajoute un certain nombre d'individus décédés à des dates et en des lieux non connus. Dans ce second groupe, ces individus présents dans l'île à la fin de l'année 1785 ont quitté Belle-Île-en-Mer après 1786, départs soit confirmés par des données de l'état civil soit établis par déduction en raison du silence des registres d'état civil bellilois. Leur nombre s'élève à 98 individus ${ }^{69}$.

Comparer les 356 départs des deux premières décennies et les 224 présences dans l'île au 31 décembre 1785 serait totalement dénué de sens. En effet, le décompte des présents tient compte des décès et des naissances survenus dans lîle pendant ces vingt années alors que le décompte des décès et des naissances survenus hors de l'île, durant la même période, n'est pas réalisable sur la base des données que nous avons été en mesure de recueillir. On peut cependant se faire une idée assez proche de la réalité si la comparaison se base non sur les individus mais sur le devenir des 78 familles installées à Belle-Île-en-Mer en 1765. En nous basant sur nos données de l'état civil, il est possible d'inventorier les familles dont les membres sont en majorité demeurés définitivement à Belle-Ille-en-Mer après 1785 en prenant comme critères les dates et les lieux de décès et de sépultures dans l'île. Au total, dixneuf familles ont vu la majorité de leurs membres demeurer définitivement à Belle-Île-en-Mer soit 24,3 \% (19/78) ${ }^{70}$. L'intégration économique et sociale de ces familles était réelle. Sur le plan économique, quatre de ces familles avaient été en mesure d'acheter cinq concessions acadiennes mises en vente entre 1777 et $1784^{71}$. Ajoutons que la succession de l'afféagiste $n^{\circ} 14$ s'élevait en 1772 à 650 livres et celle de l'afféagiste ${ }^{\circ} 2$ à 1288 livres en $1785^{72}$. Marie Françoise Granger (famille 9), Anselme Leblanc (famille 14), Laurent Babin (famille 66) exercent à Le Palais des professions commerciales. Sur le plan de l'intégration sociale rappelons que 85,7 \% des mariages conclus entre 1765 et 1785 furent des mariages mixtes.

68. Ibidem, p. 72 à 76 et annexe v, p. 122-172.

69. Ibid., p. 76 à 78 et annexe v, p. 122-172.

70. Ibid., tableau XVI, p. 80.

71. Arch. notariales de Jean Baptiste Thébault, Arch. dép. du Morbihan, série 6 E 15478 à 15484 (1775-1785).

72. CÉRINO, Christophe, op. cit., (Arch. dép. du Morbihan B 2155 et B 2167). 
Pour établir le décompte des familles acadiennes dont les membres sont majoritairement restés en France, il nous faut tout d'abord établir précisément le nombre des familles dont les membres sont en majorité partis pour la Louisiane en 1785 et ca 1785 . Ce nombre s'élève à 22 familles $^{73}$. Aucune de ces familles ne comportait de conjoint non acadien. Pour 18 de ces familles nous avons connaissance des actes notariés de vente, d'affermage ou d'exponse. Pour établir des pourcentages, seules les familles acadiennes constituées en 1785 sont à prendre en compte. Nous écartons la famille $\mathrm{n}^{\circ} 72$ originaire d'Alsace et nous soustrayons quatre familles dissoutes par décès ${ }^{74}$. Les 73 familles acadiennes se répartissent alors ainsi : 18 familles demeurées définitivement à Belle-Île-en-Mer soit 24,7 \%, 22 familles parties en Louisiane soit 30,1\%, 33 familles [ $73-(18+22)$ ] soit 45,2\% parties de Belle-Île-en-Mer et dispersées majoritairement dans différents ports bretons. Ces résultats permettent d'accorder sa juste valeur à l'émigration vers la Louisiane en 1785 et ca 1785 . Elle a concerné 30,1 \% des familles et $20 \%$ des individus installés à Belle-Île-en-Mer en 1765. Le plus intéressant en est le corollaire : 51 familles acadiennes installées à Belle-Île-en-Mer (18 + 33) sur 73 soit $69,8 \%$ (plus des deux tiers) sont restées en terre de France après 1785 .

Pour conclure, faire cohabiter deux populations à fortes caractéristiques identitaires peut se présenter a priori comme une gageure à l'issue incertaine. Dans le cas des populations belliloise et acadienne les prévisions des Cassandre ne se concrétisèrent pas. En effet, les marqueurs identitaires communs étaient appréciables : ruralité impliquant les mêmes activités agricoles, pratique religieuse catholique, respect du système monarchique de droit divin, liens familiaux et solidarité de groupe ${ }^{75}$. Les autorités mirent en œuvre des mesures propres à favoriser l'intégration des Acadiens : afféagements, dispersion des familles dans les villages, octroi d'indemnités journalières et au départ d'un pécule, de bétail et d'outils agricoles. Les bénéficiaires de ces mesures furent considérés par les Bellilois comme des privilégiés et il y eut des contestations. Mais le marqueur identitaire majeur qui pouvait s'opposer à l'intégration paraissait être la langue bretonne des ruraux bellilois. Nous avons vu que nombre d'entre eux comprenaient le français.

La quantité et la fiabilité des données factuelles et numériques collectées, nous permettent de proposer un nouvel éclairage non contestable de la question de l'intégration sociale et économique des Acadiens établis à Belle-Île-en-Mer. Entre 1765 et 1785, les mariages mixtes représentaient $85,7 \%$ des unions, ce qui témoigne de la volonté d'intégration des parents. Le rachat de concessions de partants par des Acadiens restant sur l'île,

73. MOREAU, Jean-Paul, op. cit., tableau XVII, p. 81.

74. Familles $\mathrm{n}^{\circ} 19,32,39$, et 54 .

75. CÉRINo, Christophe, « Les Acadiens à Belle-l̂le-en-Mer : une expérience originale d'intégration..." ", art. cit. 
des inventaires après décès, l'exercice de professions commerciales témoignent de la concrétisation des potentialités d'intégration économique. Près de vingt-cinq pour cent (24,7\%) des familles sont demeurées définitivement dans l'île, 30,1 \% sont parties pour la Louisiane après avoir vendu ou affermé leur concessions, enfin 45,2 \% sont restés dans des ports bretons. Les départs pour des raisons économiques certaines ne dépassent pas sept. Près de soixante-dix pour cent $(69,8 \%)$ des familles acadiennes sont restées en France. L'expérience de Belle-Île-en-Mer ne saurait être qualifiée d'échec et la " légende noire " évoquée par Dominique Guillemet ne relève en réalité que d'une erreur de jugement historiographique.

\section{RÉSUMÉ}

En 1765, 78 familles de 363 réfugiés acadiens furent établies à Belle-l̂le-enMer. L'historiographie insiste très tôt sur l'échec de cette implantation et, par la suite, de très importantes divergences statistiques se sont opposées à une vision claire et précise du devenir de cette population acadienne. L'objectif majeur de notre travail a été d'établir des données factuelles et numériques dûment vérifiées et validées et aussi exhaustives que possible. Ceci nous a permis de proposer un nouvel éclairage sur le devenir social et économique des Acadiens de Belle-Île-en-Mer. En dépit des divergences identitaires, 86,7 \% des mariages furent des mariages mixtes; $24,7 \%$ des familles acadiennes restèrent définitivement dans l'île; 30,1 \% partirent en Louisiane et 45,2 \% s'établirent dans différent ports du littoral breton.

\section{ABSTRACT}

In 1765, 78 families of 363 Acadians refugees were established in Belle-Île-enMer. From the very start, historiography emphasised the failure of this implantation but statistical differences of great importance challenged any simplistic vision of the subsequent outcomes for the Acadian population. The major objective of our work was duly to verify and validate the factual and digital data and render it as exhaustive as possible. This allows us to shed new light on the social and economic future of these Acadian refugees. In spite of their differences of origin, 86.7 per cent of the marriages were mixed marriages, 24.1 per cent of families stayed permanently on the island, 30.1 per cent left for Louisiana and 45.2 per cent became established in various harbours of Breton coast. 